\title{
Enhanced Artificial Bee Colony method for node deployment and routing in Wireless Sensor Networks
}

\author{
Basavaraj Madagouda ${ }^{1}$, Dr. R. Sumathi ${ }^{2}$ \\ ${ }^{1}$ Department of Computer Science and Engineering, Jain College of Engineering and Research Belagavi, VTU \\ Belagavi, India
}

${ }^{2}$ Department of Computer Science and Engineering, Siddaganga Institute of Technology Tumkur, VTU Belagavi, India

Article Info

Volume 7, Issue 6

Page Number: 28-37

Publication Issue :

November-December-2021

\section{Article History}

Accepted : 01 Nov 2021

Published : 10 Nov 2021

\section{ABSTRACT}

Wireless networks provide small sensing, machine and wireless networking nodes. Different designs and implementation techniques were built based on the device requirements for wireless network sensors (WSN). Sensor networks are used in various applications, such as environmental monitoring, home automation, military applications, etc. In this study introduce an architectural survey and deployment of nodes in the Wi-Fi Sensor network in this article. The environmental features that can be added to the sensor networks are given. The program relies on the node installed in the WSN and is deterministic or random. But the biggest issue in both cases is the coverage of the region involved. Researcher also describe WSN routing protocols. In this paper, a new technique to deployment problem is proposed based on the artificial bee colony (ABC) algorithm which is enhanced for the deployment of sensor networks to gain better performance by trying to increase the coverage area of the network and energy consumption. The good performance of the proposed EABC algorithm shows that it can be utilized in the deployment of WSN.

Keywords: Node Deployment, Routing, Artificial Bee Colony, Wireless Sensor Networks.

\section{INTRODUCTION}

Wireless network is made up of a wide variety of sensor nodes; wireless sensor communications. A variety of applications, including industrial sensor network, volcanic detection networks, climate control, health surveillance, domestic automation is suggested [1]. Internal organisation of software and hardware can help it operate efficiently and rapidly to meet changing demands, needs and applications. Autonomy and adaptability are the key aspects to remember. Autonomy states that sensors may be used in an unattained region or in a physically inaccessible environment and that they are therefore expected by the basic stations or human managers to work as little as possible [2]. Sensor networks should facilitate 
adaptation to the sensing way sensors operate, for example sensors must decrease processing cycles in a manner that reduces the sensor's voltage usage in order to optimise the use of sensor energy if sensor readings do not shift dramatically [3]. In several sensor network implementations, mobility is involved. Coordination in sensor networks primarily relies on how the data is collected and the applications are collected, mostly on the activities of data transferred to nodes and data collected by environmental nodes. In the WSN, the use of nodes remains the major problem. The node implementation plan is built based on applications.

\section{Wireless Node Sensor Systems}

In Figure 1, shows the architecture for wireless node sensor systems, consisting of four major subcomponents (1) a sensor subsystem which has an or more sensors for data collection (2) the wireless data transmission subsystem; (3) the data memory and microcontroller computing subsystem; and (4) the power supply device.

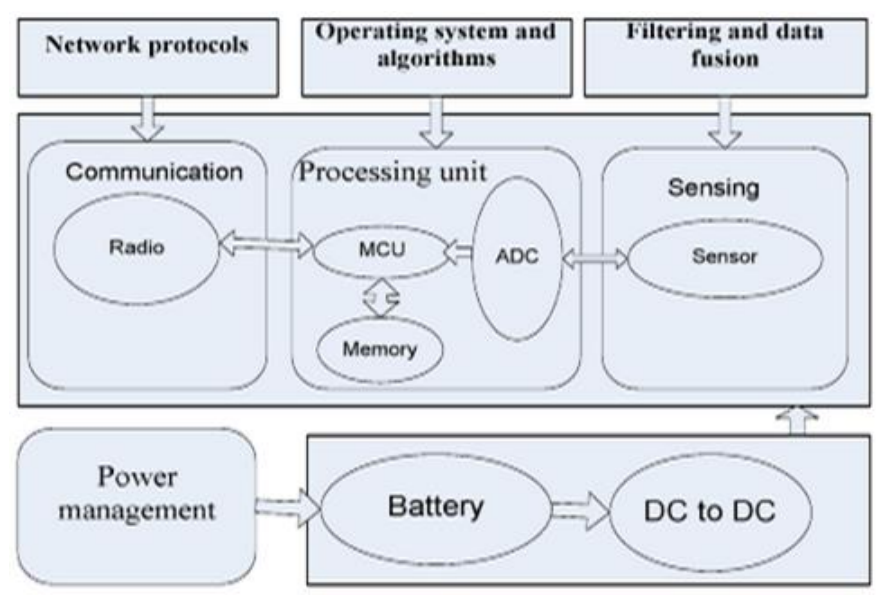

Fig.1. Sensor Node System Architecture

The power disturbance depends, of course, heavily on the individual node type. The stargate sensor node has demonstrated that its power characteristics differ from that of a Mote type sensor node. The sensor includes a variety of power sources including signals sampling, delivery of training signals to electricity and signal conditioning.

\section{Artificial Bee Colony (ABC) Algorithm}

Artificial Bee Colony (ABC) Algorithm Model The standard $A B C$ algorithm is a population-based optimization algorithm. The working principle of $\mathrm{ABC}$ is as illustrated in Figure 2. Based on the figure, the working principle of $\mathrm{ABC}$ can be categorized into five main phases which are initialization, employedbees, onlooker-bees, scout-bee, and termination phases which consist of a total of twelve stages or processes.

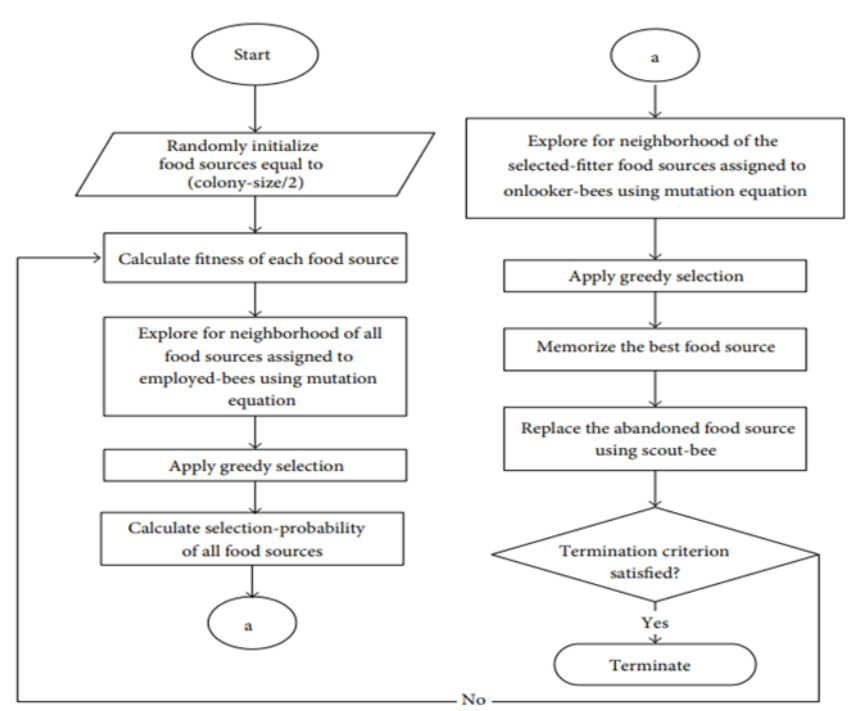

Figure 2. Flowchart of standard Artificial Bee Colony Algorithm

In $\mathrm{ABC}$, three phases are performance-deciding phases which are employed-bees, onlooker-bees, and scout-bee phases while the other two are supporting phases. The exploration process of the algorithm takes place in employed-bees and onlooker-bee's phases where the bees need to explore the neighbourhood of the food sources allocated to them. Meanwhile, the exploitation process happens in the onlooker-bees phase when onlooker-bees apply fitness-proportion selection scheme in order to select the selected-fitter food sources. The details of the phases are discussed in 
the following subsections and more details of $A B C$ can also be found in [18].

\section{Problem Formulation}

The key problems in the node implementation techniques include: 1) The energy hole problem; where data streams follow a "many-for-one" mode which subject's nodes near the Sink to greater traffic load, resulting in premature energy depletion and the creation of an "energy hole" around the Sink 2) node coverage area, locating the dead nodes in the area of the coverage and the nodes on the borders of the other nodes. As a result, more focus was paid to methods of implementation of nodes with and without restrictions dependent on and in general applications. 3) The communication overhead problem; because the self-energy of sensor nodes are limited, there is control overhead regardless of the routing protocol algorithm and thus an inherent need to control the energy consumption of network nodes;

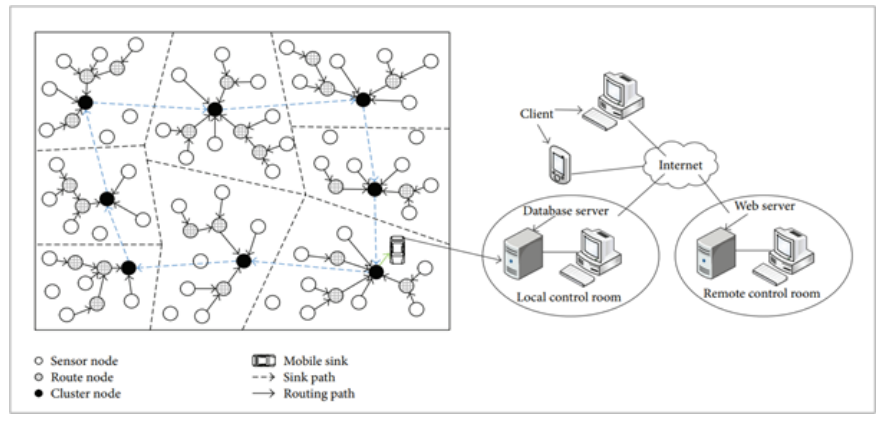

Figure 3. Mobile Wirelesses Sensor Network

Architecture

Previous researchers have addressed the above problems by means of node mobility. The mobile node acts as a data collector, migrating through the network in accordance with defined routes to conduct data collection in the monitoring area. By taking advantage of mobile node features, the connectivity, coverage, and energy distribution of mobile wireless sensor networks (MWSN) can be deployed dynamically or adjusted according to real- time conditions so as to fill in routing voids in the network and blind zones in the sensor.

Mobile Sinks can significantly reduce network energy consumption and avoid the energy hole caused by multihop transmission so that data collection is unaffected even if there is no data path between nodes. Mobile Sinks have particularly obvious advantages in sparse or unconnected networks. Nevertheless, because the speed of the moving sink node cannot be compared to the speed of wireless transmission, there is a delay from generation to transmission of sensor data. Figure 1 shows an example of a typical MWSN data collection process, where first the monitoring area is clustered and then the sensor node transmits the collected data to the cluster head node via routing for buffer memory and the mobile Sink only needs to visit the cluster head nodes to realize data collection, so the moving path is truncated, data transmission latency is reduced, and the network data collection efficiency and reliability are improved compared to a network without a mobile Sink.

Verified through extensive analysis, this paper presents an efficient and reliable data collection mechanism, a mobile wireless sensor network (MWSN) data collection program based on the enhanced artificial bee colony algorithm (EABE). The main contributions of this paper can be summarized as follows: (1) The mobile Sink data collection process, cluster head selection problems, routing path from ordinary nodes to the cluster head node, and mobile Sink path optimization, when considered synthetically. (2) The path optimization of the mobile sink can be formulated as a traveling salesman problem; then the artificial bee colony algorithm can be used to seek the features of the optimal soluti6on and the shortest path of the mobile Sink so as to improve network data collection efficiency. (3) Extensive numerical results are provided below 
which demonstrate the usage and efficiency of the proposed data collection algorithm.

The rest of the paper is organized as follows. In Section 2, we define the literature review and related work done so far. Section 3 explores proposed solution and EABC algorithm to solve the problem for sensor networks. The efficacy of our solution techniques through extensive simulation and analyse the results is shown in Section 4. Finally, we conclude the paper with a direction for future work in Section 5.

\section{Literature Survey}

P. Gajbhiye and A. Mahajan at el in [10] discussed and evaluated core issues relevant to the fundamental implementation of the sensor wireless network. They separated nodes into two classes, namely random, regulated or determinist. The node is organised to achieve networking topology and planned network goals in controlled approaches. Though node substitution is a key consideration for network success in random and dynamic roll-outs. This survey concludes that static methods are more simple, but that the controlled implementation of the node is no challenge. Finally, authors conclude that deterministic methods are more useful than the use of random nodes.

It is a challenge to identify the best Wireless Sensor Node (WSN) distribution approach to avoid costs, resist node crashes, reduce computers and connectivity overheads, and preserve high coverage and network links. Indeed, since they can directly affect network life and service, sensor coverage and network compatibility are two of WSNs' main issues. For WSN coverage, S. Birtane, S. Kazdal and O. K. Sahingoz at el in [11] assume optimal conditions for connectivity. Most versions of this problem are (NPcomplete), and approximation algorithms for certain versions of polynomial time cannot be built until $\mathrm{P}=$
NP. For some of the versions authors also establish a heuristic strategy, such that comprehensive simulations can test the effectiveness of the heuristic. They still want to determine whether a path can be found over a certain WSN topology between several nodes. This is used as a connectivity indicator of a network coverage. Therefore, the requirements for communication and converging over a clustered system in WSNs are required and necessary. Then, authors present a dynamic programming analysis using queuing network modelling methods on connections with a cluster structure coverage and its impact on routing in widespread WSNs. The assessment of the efficiency of the proposed networks indicates that the availability of nodes, the coverage of sensor nodes and the links were adequately improved to optimise network life.

J. N. Al-Karaki and A. Gawanmeh at el in [12] introduces and measures an algorithm known as DeVForce-AP for wireless sensor node deployment. Their suggested algorithm incorporates the advantages of the triangulation technique (D) and the expanded virtual power (eVForce) algorithm using the adaptive parameter tuning method (AP). DeVForce-AP aims to enhance its sensory coverage and network life with its heterogeneous wireless sensor node. Convenient topics such as environmental barriers, network access and the sensing industry have also been addressed. There are two phases in DeVForce-AP; Delaunay Triangulation Approach is used by DeVForce-AP to identify and delete the largest coverage hole within the field of interest in the first step. In the second step, DeVForce-AP applies an expanded algorithm for virtual force to prevent environmental obstructions from occurring and preserve network connectivity. In addition, the DeVForce-AP suggested changes the parameters dynamically to the environmental situation. Thus, DeVForce-AP parameters should not be configured. The findings of the simulation indicate that the DeVForce-AP proposed outperforms the 
altered application, conventional Delaunay triangulation and the traditional approaches used for virtual entities.

WSN represent the number of arbitrarily distributed nodes with static environmental monitoring location. A special hierarchy technique is applied to gather the origins of knowledge within the static WSN called a graded node. The lowest cost and energy efficient route must be established to move data to a drain. The nodes need to be installed and the graph designed to deliver network traffic to sinking in multipath ways to reach an energy efficient route. The suggested implementation strategy by R. Kuawattanaphan, P. Champrasert and S. Aramkul in [13] for the device and grade nodes is applied in order to accomplish the above network parameters and to hit the sink node via a minimal hop number and to match the energy of the nodes while extending the existence of the network. In addition to improving service efficiency, an energy saved technology is built with the MAC layer and the physical wireless layer and criteria differ to minimise delays and energy usage. Studying the simulation findings and interpretation reveals that the model deployed improves network life substantially. Therefore, this device provides a great cost-effective and energy-efficient approach to deploy and route nodes into large WSN for longer time monitoring.

Daozong Sun, Weixing Wang, Jianqing $\mathrm{Lu}$ and Zuanhui Lin et al in [14] proposes an ecosystem surveillance system for a tea plantation with WSN node and portal node. Each sensing node consisted of the microprocessor ATmega 128L and the transceiver module CC2420. As a hardware platform kernel in gateway node, S3C2410X was used as a microcontroller. In laboratory and tea plantation studies with the MESH routing protocol networking was performed to test network performance at various network sizes and different types of node deployment. The findings illustrate that the temperature and humidity of the tea plantation can be obtained by the device and sent remotely to the base station. A successful solution for environmental monitoring of complex geographical situations such as tea planting has been investigated, and can be taken as an example of an in-field measurement solution.

B. Diribu and M. Libsie et al in [15] proposes an alternative to node deployment and forecasts excellent numbers of nodes in sense of a certain agriculture work. A minimum number of sensor nodes forming a sensor network is recommended for a deployment framework. Based on various success indicators, the proposed framework was tested. A PEGASIS routing protocol has also been chosen because it is faster than LEACH. Based on assessment observations, the current implementation approach (i.e. scheme) for nodes in wireless sensing networks is a safer alternative for precision agriculture (WSNs). It can be used for any form of the farm area monitored. Proposed method can also be employed for precision agriculture for other related uses, including largescale environmental monitoring.

In order that location error is effectively minimised, C. Hsiao, Y. Tsai and W. Zheng et al in [16] concentrates on implementing WSNs for SBL algorithms. First, they build a toolkit for the site location for server deployment to evaluate the effects of deployment node positions. Then a new strategy for distributing nodes, based on the observation using the toolkit is developed. Maker considers several criteria to decrease location error by using sensor nodes. (1) Standard polygonal area deviations sliced by sensor node perpendicular bisectors should be maintained as small as possible, (2) distance should be kept between the sensor nodes, and (3) angles should be optimised between perpendicular bisectors. For assessment, authors use the network simulator ns2. The effects of the ns2 simulation show that their proposed solution to implement the node theoretically decreases the SBL location error. 
In paper [17] authors D. Izadi, J. Abawajy and S. Ghanavati addressed the topic of coverage of electrical effectiveness WSN. They formulated the problem and showed that it was not fully resolved in this literature, especially for random deployment model. They also proposed a self-healing algorithm based on the type-2 flooded logic systems to maintain the coverage rate of the SCN. They have shown, from an experimental analysis, that the approach proposed is effective in retaining both the coverage ratio of the solution and energy utilisation in contrast with several recent solutions.

P. Chatterjee, S. C. Ghosh and N. Das at el [18] proposed a novel graded node implementation technique that produces minimal traffic, just enough for coverage, to capture data streams in static WSN. A distributed load-balanced data collection algorithm is built on the basis of this node delivery to send packets to the sink node through minimum-hop routes, which also helps restrict network traffic in turn. On the basis of a possible lower node number limit, a mathematically average case test is based on optimal matches in random bipartisan maps. Studies and simulation studies demonstrate that, in the proposed model, the network life is substantially increased and the costs of unnecessary usage are drastically overridden. This method thus represents a very effective and cost-efficient deployment of nodes and long-term strategy on routing through large WSN.

In order to ensure the maximisation of the WSN coverage for efficient tracking, a sensor node placing scheme based on TPSMA has been proposed. Zainol Abidin, Husna \& Din, Norashidah at el in [19] demonstrates the network model which consists of many places controlled of equal width. Based on the results obtained from a simulation, it can be inferred that WS $\mathrm{N}$ with the TPSMA sensor nodes full coverage system provides a slightly higher coverage and a lower standardisation than minimax and lexmin systems. This results in WSN deployed in the proposed scheme to have a longer lifetime coverage. A multi-objective positioning scheme for TPSMA sensor nodes will be further developed.

\section{System Architecture}

The Enhanced Artificial Bee Colony (EABC) algorithm is a swarm intelligence method inspired by the intelligent foraging behaviour of honey bees, is used for the dynamic deployment problem of WSNs. The aim of the optimization technique is to maximize the coverage rate of the network. In the network's scenario, it is assumed that:

- The detection radii of the sensors are all the same (r).

- All of the sensors have the ability to communicate with the other sensors.

- $\quad$ All sensors are mobile.

In the EABC algorithm, the position of a food source represents a possible solution to the optimization problem and the nectar amount of a food source corresponds to the quality of the associated solution. Therefore, the deployment of the sensors in the sensed area refers to a food source in the algorithm. The coverage rate of the network, i.e. the total coverage area, corresponds to the fitness value of the solution. In the EABC model, artificial bee colonies, in which the goal of the bees is to find the best solution, comprise 3 groups of bees:

1. Employed bees: They are associated with a particular food source which they are currently exploiting or are "employed" at. They carry with them information about this particular source, its distance and direction from the nest, the profitability of the source and share this information with a certain probability.

2. Onlookers: A forager bee who tries to find a food source by means of the information given by the employed bee is called Onlooker. 
3. Scouts: A forager bee that looks for a food source by searching the environment randomly to exploit is called a scout.

In the proposed system first, we initialise the network and the best paths are stored. After the best path has been stored the employed bees are send in order to find out the change in paths or any link breakages. The information gathered by Employed bee is analysed and then the onlooker bee is sent to the nodes discovered by employed bees. The Onlooker bees collect the details of the nodes already visited. They give status of nodes. Depending on the information from the onlooker bees the best paths are determined. The Scout Bees perform random search for nodes. In this system we have sensor nodes which are mobile and the shortest path is calculated by the nodes depending on the RSSI value, distance between the nodes, number of hopes between the nodes.

\section{A. Calculation of RSSI}

Suppose that $R S S I_{(i, j)}$ is a signal strength value, which was send from node $j$ to node $i$, and measured from the node $i$. Then

When $D_{o}=1 \mathrm{~m}$,

$$
R S S I_{(i, j)}=A-10 n \log \left(D_{i j} / D_{o}\right)
$$

$$
D=10^{A-R S S I_{(i, j)} / 10 n}
$$

The unit for $R S S I_{(i, j)}$ is $\mathrm{dBm} . D_{i j}$ is the actual distance between node $i$ and node $j$. The radio parameter $A$ is defined as the absolute value of the average power in $\mathrm{dBm}$ received at a close-in reference distance of one meter from the transmitter, assuming an omni directional radiation pattern. The radio parameter $\mathrm{n}$ is defined as the path loss exponent that describes the rate at which the signal power decays with increasing distance from the transmitter The parameter A and $n$ can be estimated empirically by collecting RSSI data (and therefore path loss data) for which the distances between the transmitting and receiving devices are known. The typical value $A$ is in the range $[30.0,50.0]$ with precision 0.5. A typical value for $\mathrm{n}$ is 2 to 4 .
B. Enhanced Artificial Bee Colony Algorithm (EABC)

Initialize Population Repeat

- $\quad$ Place the employed bees on their food sources

- Place the onlooker bees on the food sources depending on their nectar amounts

- $\quad$ Send the scouts to the search area for discovering new food sources

- Memorize the best food source found so far until requirements are met

The search consists of three steps: moving the employed and onlooker bees onto the food sources and calculating their nectar amounts and determining the scout bees and directing them onto possible food sources. A food source position represents a possible solution to the problem to be optimized. The amount of nectar of a food source corresponds to the quality of the solution. Onlookers are placed on the food sources by using a probability-based selection process. As the nectar amount of a food source increases, the probability value with which the food source is preferred by onlookers increases. The scouts are characterized by low search costs and a low average in food source quality. One bee is selected as the scout bee. The selection is controlled by a control parameter called "limit". If a solution representing a food source is not improved by a predetermined number of trials, then that food source is abandoned and the employed bee is converted to a scout.

\section{Result}

\section{A. Experimental Setup}

Table 1 shows the simulation parameter setup used for experimental analysis of EABC algorithm with $\mathrm{ABC}$ and Random Walk Algorithm.

Table 1. Simulation Parameters

\begin{tabular}{|c|c|}
\hline Routing Protocol & $E A B C$ \\
\hline Number of Nodes & 30 \\
\hline Area size & $1000 \mathrm{~m} \times 1000$ \\
\hline
\end{tabular}




\begin{tabular}{|c|c|}
\hline & $m$ \\
\hline MAC & $802.11 \mathrm{~b}$ \\
\hline $\begin{array}{c}\text { Radio Frequency } \\
\text { Range }\end{array}$ & $250 \mathrm{~m}$ \\
\hline $\begin{array}{c}\text { Simulation Time } \\
\text { Traffic Source }\end{array}$ & $6 \mathrm{sec}$ \\
\hline Packet Size & $512 \mathrm{~KB}$ \\
\hline $\begin{array}{c}\text { Mobility model } \\
\text { speed }\end{array}$ & $5 \mathrm{~m} / \mathrm{s}$ \\
\hline Initial Energy & 0.5 Joules \\
\hline
\end{tabular}

\section{B. Energy Consumption.}

Energy consumption is an important indicator of network performance. The total energy consumption of the three network algorithms is shown in Figure 4. As the number of simulation iterations increased, network energy consumption gradually increased for all three though random walk showed the largest increase in energy consumption, followed by the $A B C$ algorithm and finally the proposed algorithm EABC, which saved $19.1 \%$ energy compared to random walk and $3.5 \%$ energy compared to $\mathrm{ABC}$. We next simulated the same scenarios but iteratively increased the number of nodes $(100,200,300, \ldots, 1000$ sensor nodes) in a $1000 \mathrm{~m} \times 1000 \mathrm{~m}$ field, as shown in Figure 4 .

\section{ENERGY CONSUMPTION COMPARISON GRAPH}

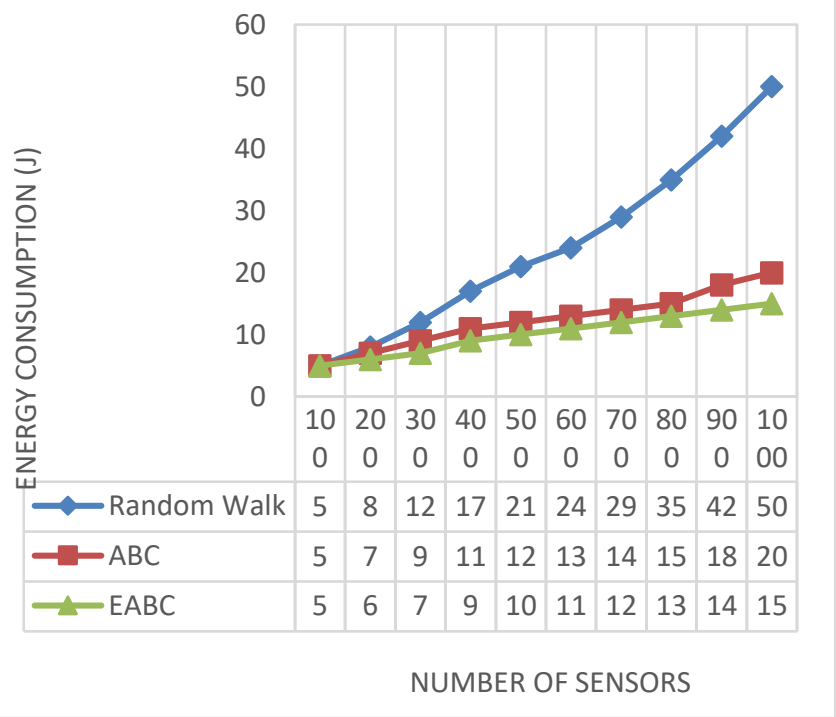

Figure 4. Energy consumption comparison graph
As the number of nodes increased, the energy consumption of all three algorithms gradually increased, but again, random walk consumed the most energy; and again, the $\mathrm{EABC}$ algorithm consumed the least energy, while the $\mathrm{ABC}$ algorithm performed second best.

\section{Network Latency}

We used the transmission delay (i.e., latency) of successfully received data packet to measure the realtime performance of various protocols. Data transmission time at the source node was recorded as $T s$, the reception time at the Sink node was recorded as $\operatorname{Tr}$, and average transmission delay was calculated as follows:

$$
T_{\text {trans }}=\frac{1}{N_{r}} \sum_{i=1}^{N_{r}}\left(T_{r i}-T_{s i}\right),
$$

where $N r$ is the total number of successfully received packets.

\section{NETWORK LATENCY COMPARISON GRAPH}

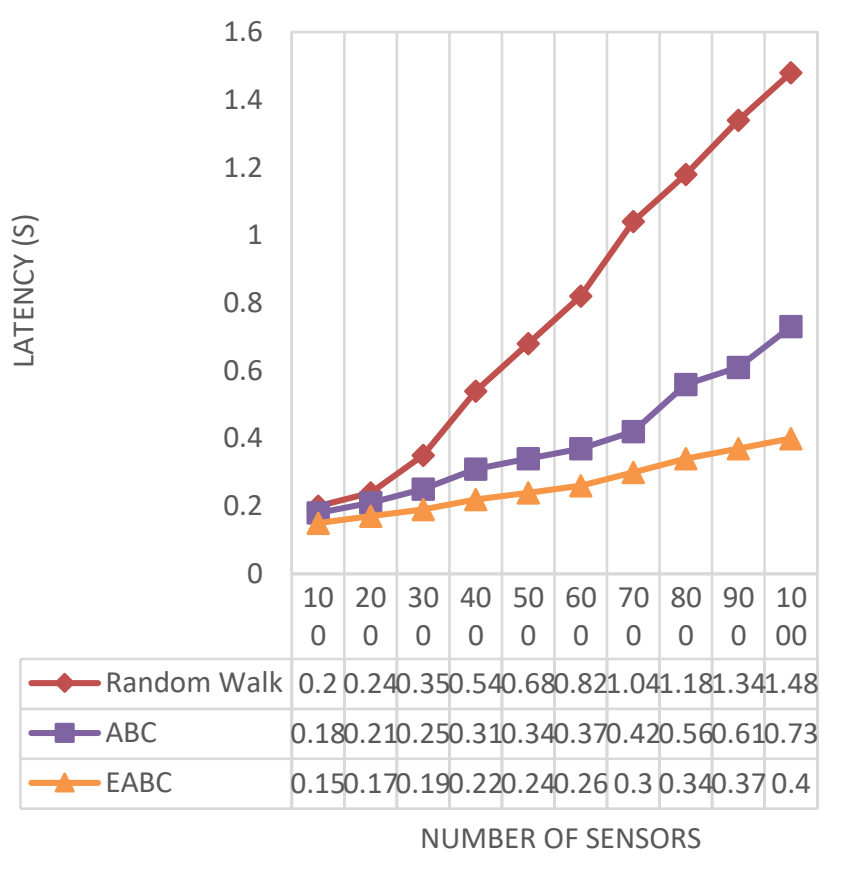

Figure 5. Network Latency Comparison Graph 
The network latency of the three algorithms is shown in Figure 5, as expected, random walk showed the largest delay (nearly 2 s) initially which then gradually decreased. The latency of EABC and ABC algorithms was initially relatively large, around $0.8 \mathrm{~s}$, mainly because swarm intelligence algorithms are random during the first iterations while searching the optimal path. After subsequent learning and optimization, the final delay of $A B C$ was about $0.2 \mathrm{~s}$, while that of EABC algorithm was about 0.1 s.

\section{Conclusion}

In this paper researcher addressed state-of-the-art architectures and emerging techniques in the wireless sensor system for node implementation. It also mentioned the environmental characteristics added by the sensor networks. In sensor networks, there are so many problems that need to be overcome. We propose an Enhanced Artificial Bee Colony Optimization Algorithm (EABC), which uses Received Signal strength to calculate the distance to consider as a routing metric. The use of location information as a heuristic parameter resulted in a significant reduction of the time needed to establish routes from a source to a destination which is important for a reactive routing algorithm. In this study, the Enhanced ABC algorithm is applied to the dynamic deployment problem in WSNs with mobile sensors. We can also apply the EABC algorithm for dynamic deployment of WSNs including not only mobile sensors but also stationary ones.

\section{REFERENCES}

[1]. Lakshman Krishnamurthy, Robert Adler, Phil Buonadonna, Jasmeet Chhabra, Mick Flanigan, Nandakishore Kushalnagar, Lama Nachman, and Mark Yarvis, "Design and deployment of industrial sensor networks: experiences from a semiconductor plant and the north sea," Proc. The 3rd international conference on Embedded networked sensor systems (SenSys '05), pp. 6475, ACM Press, 2005.

[2]. Geoffrey Werner-Allen, Konrad Lorincz, Mario Ruiz, Omar Marcillo, Jeff Johnson, Jonathan Lees and Matt Welsh, "Deploying a Wireless Sensor Network on an Active Volcano," Special Sensor Nets issue of IEEE Internet Computing, 2006.

[3]. Ning Xu, "A Survey of Sensor Network Applications," University of Southern California,

http://enl.usc.edu/ $\sim$ ningxu/papers/survey.pdf, 2003.

[4]. Stefan Dulman, Paul Havinga "Architectures for Wireless Sensor Networks", IEEE ISSNIP 2005.

[5]. Pruet Boonma, Paskon Champrasert, Junichi Suzuk"BiSNET: A Biologically-Inspired Architecture for Wireless Sensor Networks", IEEE 2006.

[6]. Karim Seada, Ahmed Helmy"Rendezvous Regions: A Scalable Architecture for Service Location and Data-Centric Storage in LargeScale Wireless Networks" in the proceedings of the 18th International Parallel and Distributed Processing Symposium (IPDPS'04), IEEE 2004.

[7]. T. Clouqueur, V. Phipatanasuphorn, P. Ramanathan and K. k. Saluja, "Sensor Deployment Strategy for Target Detection," WSNA, 2002.

[8]. S. Dhillon, K. Chakrabarty and S. Iyengar, "Sensor placement for grid coverage under imprecise detections," Proc.International Conference on Information Fusion, 2002.

[9]. Kushwaha, Sumit. (2011). node architecture and its deployment in wireless sensor networks: a survey. 10.13140/2.1.2616.9283.

[10]. P. Gajbhiye and A. Mahajan, "A survey of architecture and node deployment in Wireless Sensor Network," 2008 First International Conference on the Applications of Digital Information and Web Technologies 
(ICADIWT), Ostrava, Czech Republic, 2008, pp. 426-430, doi: 10.1109/ICADIWT.2008.4664386.

[11]. S. Birtane, S. Kazdal and O. K. Sahingoz, "2D coverage analysis of sensor networks with random node deployment," 2017 International Artificial Intelligence and Data Processing Symposium (IDAP), Malatya, 2017, pp. 1-5, doi: 10.1109/IDAP.2017.8090343.

[12]. J. N. Al-Karaki and A. Gawanmeh, "The Optimal Deployment, Coverage, and Connectivity Problems in Wireless Sensor Networks: Revisited," in IEEE Access, vol. 5, pp. 18051-18065, 2017, doi: 10.1109/ACCESS.2017.2740382.

[13]. R. Kuawattanaphan, P. Champrasert and S. Aramkul, "A Novel Heterogeneous Wireless Sensor Node Deployment Algorithm With Parameter-Free Configuration," in IEEE Access, vol. 6, pp. 44951-44969, 2018, doi: 10.1109/ACCESS.2018.2865279.

[14]. Daozong Sun, Weixing Wang, Jianqing Lu and Zuanhui Lin, "Design of WSN nodes and network performance analysis in a tea plantation," IET International Conference on Wireless Sensor Network 2010 (IET-WSN 2010), Beijing, 2010, pp. 144-147, doi: 10.1049/cp.2010.1043.

[15]. B. Diribu and M. Libsie, "Shape based customized node deployment approach for WSNs," AFRICON 2015, Addis Ababa, Ethiopia, 2015, pp. 1-5, doi: 10.1109/AFRCON.2015.7331943.

[16]. Hsiao, Y. Tsai and W. Zheng, "Node deployment strategy for WSN-based nodesequence localization considering specific paths," 2013 IEEE Eighth International Conference on Intelligent Sensors, Sensor Networks and Information Processing, Melbourne, VIC, Australia, 2013, pp. 201-206, doi: 10.1109/ISSNIP.2013.6529789.
[17]. Izadi, J. Abawajy and S. Ghanavati, "An Alternative Node Deployment Scheme for WSNs," in IEEE Sensors Journal, vol. 15, no. 2, pp. 667-675, Feb. 2015, doi: 10.1109/JSEN.2014.2351405.

[18]. P. Chatterjee, S. C. Ghosh and N. Das, "Load Balanced Coverage with Graded Node Deployment in Wireless Sensor Networks," in IEEE Transactions on Multi-Scale Computing Systems, vol. 3, no. 2, pp. 100-112, 1 April-June 2017, doi: 10.1109/TMSCS.2017.2672553.

[19]. Zainol Abidin, Husna \& Din, Norashidah. (2013). Sensor Node Placement in Wireless Sensor Network Based on Territorial Predator Scent Marking Algorithm. ISRN Sensor Networks. 2013. 10.1155/2013/170809.

\section{Cite this article as :}

Basavaraj Madagouda, Dr. R. Sumathi, "Enhanced Artificial Bee Colony method for node deployment and routing in Wireless Sensor Networks", International Journal of Scientific Research in Computer Science, Engineering and Information Technology (IJSRCSEIT), ISSN : 2456-3307, Volume 7 Issue 6, pp. 28-37, November-December 2021. Available at doi : https://doi.org/10.32628/CSEIT21767 Journal URL : https://ijsrcseit.com/CSEIT21767 